\title{
POLÍTICAS AMBIENTALES, CAMBIO CLIMÁTICO Y OPINIÓN PÚBLICA EN ESCENARIOS REGIONALES El caso de Andalucía
}

\section{ENVIRONMENTAL POLICY, CLIMATE CHANGE AND PUBLIC OPINION THE CASE OF ANDALUSIA}

\author{
EduARdo Moyano \\ Instituto de Estudios Sociales Avanzados (IESA-CSIC), Córdoba, España \\ emoyano@iesa.csic.es \\ Ángel Paniagua \\ (CCHS-CSIC), España \\ angel.paniagua@cchs.csic.es \\ Regina Lafuente \\ Instituto de Estudios Sociales Avanzados (IESA-CSIC), Córdoba, España \\ rlafuente@iesa.csic.es
}

\section{Resumen}

Este artículo es una contribución, desde la Sociología Ambiental, al estudio de las relaciones entre opinión pública y políticas ambientales. El tema que abordan los autores es la variabilidad existente en el modo en que la población percibe los problemas relacionados con el medio ambiente dependiendo del ámbito geográfico que se tome como referencia (local, regional, nacional, global), tema éste que tiene importancia para la formulación de las políticas públicas (concretamente, para la aplicación de los principios de mitigación y adaptación en la lucha contra el cambio climático). Sus autores toman la comunidad autónoma andaluza como caso de estudio, a partir de la información proporcionada por el Ecobarómetro de Andalucía elaborado, desde 2001, por el IESA-CSIC. La principal aportación de este artículo consiste en señalar cómo, ante el cambio climático, la población percibe que la política dirigida a mitigar los efectos de este problema de escala global es una especie de "suprapolítica" disociada de las acciones regionales/locales.

\section{Palabras Claves Adicionales}

Cambio climático, Conciencia ambiental, España, Opinión pública.

\section{Abstract}

The objective of this article is to contribute, from the environmental sociology, to the debate on the relationship between public opinion and environmental policies. We show the diversity existing at social perceptions on environmental issues in accordance to the geographical level we take as reference (local, regional, national and global). This topic is very important both to define and implement policies aimed to mitigate the effects of climate change. The empirical analysis has carried out of the information offered by the Ecobarómetro of 
Andalusia, which is an annual survey made from 2001 by the IESA-CSIC. The main contribution of this article is to show how, contrary to the sustainable development agenda, the population does not connect the global dimension of the climate change to the need of implementing regional/local policies. The Andalusian people perceive that the policy aimed to mitigate the effects of climate change is a "supra policy", and they consider that actions implemented at the regional/local level are none effects on global environmental problems.

\section{Additional Keywords}

Environmental Policy, Global Environmental Change, Public Opinion, Spain.

\section{INTRODUCCIÓN}

Uno de los retos de la moderna Sociología Ambiental es el análisis de las actitudes, los valores y el comportamiento de la población ante los problemas relacionados con el medio ambiente a escala global (es decir, los que afectan al conjunto del planeta, como el cambio climático, la disminución de la capa de ozono, la pérdida de biodiversidad, etc.). El objetivo de los escasos estudios existentes en este área de la investigación social es comprobar en qué medida las actuaciones de los poderes públicos influyen en los ciudadanos a la hora de valorar la gravedad de las cuestiones ambientales de escala global, y hasta qué punto el contenido de estas políticas públicas refleja el modo en que la población percibe los problemas globales del medio ambiente.

A diferencia de lo que ocurre con los problemas ambientales más circunscritos a sectores concretos o ámbitos territoriales específicos (contaminación de mares y ríos, calidad del agua, ruido, eliminación de residuos urbanos, etc.), no se han estudiado todavía con suficiente rigor desde la Sociología Ambiental la percepción social de los problemas de escala global y las actitudes de la población ante ellos. Por ello, este asunto constituye una notable oportunidad para el futuro desarrollo de esta disciplina (Seacrest, Kuzelka y Leonard, 2000). En el ámbito político, la mayor o menor gravedad con que los ciudadanos perciben los cambios producidos en el medio ambiente a nivel global y el grado de importancia que otorgan a su resolución —o al menos a la necesidad de mitigar sus efectos- son asuntos de gran relevancia. Es un hecho que el éxito de muchas de las acciones públicas destinadas a luchar contra tales efectos depende de la implicación de la ciudadanía, y eso varía según cómo los perciba y valore la población en cada territorio. Por ello, estos temas constituyen un área relevante de estudio, con múltiples implicaciones (tanto de tipo espacial, como temporal) para la implementación de las políticas ambientales dirigidas a tratar de mitigar los problemas de escala global. Es sobradamente conocido el punto de vista que considera de gran importancia para el estudio de los problemas ambientales conocer las actitudes, los valores y el comportamiento de la población, dadas las diferencias observadas en el modo como estos problemas son percibidos por los individuos, según el nivel de estudios, la edad, el sexo, el hábitat de residencia o la escala geográfica que se tome como referencia (Cohen et al., 1998). De acuerdo con este planteamiento cabe pensar, respecto a los problemas ambientales de escala global, que si los individuos perciben de modo diferente su 
gravedad (ya sea por razones sociodemográficas o por la forma en que tales problemas se manifiestan en cada espacio territorial o ámbito geográfico), la consecuencia más directa para el análisis sería aceptar la existencia de distintos puntos de vista en el debate ambiental y admitir que éste es un asunto de notable complejidad.

De tal diversidad de actitudes y percepciones sociales se derivaría también el hecho de que las actuaciones públicas en materia de cambio climático sean, en sí mismas, actuaciones marcadas por la incertidumbre de sus efectos, tanto en lo que se refiere a las políticas de adaptación, como a las de mitigación, dado que el éxito de tales políticas depende, en gran medida, del grado de receptividad y colaboración que encuentren en la población (Dessai, O’Brien y Hulme, 2007; Paniagua, Moyano, 1998; Tabara, 2003). Además, hay que tener en cuenta que, si bien las políticas ambientales se formulan y aplican en el marco de estructuras institucionales bien definidas (ministerios de medio ambiente o departamentos similares), la realidad es que sus orientaciones, contenido y desarrollo son fruto, en última instancia, del conjunto de valores, creencias, normas y expectativas sociales que predominan en una sociedad concreta y en un momento histórico determinado (O'Riordan y Jordan, 1996). Más recientemente, y con ocasión de los debates en torno al cambio climático, algunos estudios señalan el carácter socialmente construido de los discursos y disputas políticas que surgen en torno a estos asuntos (Pettenger, 2007; Sunderly, 1995).

La política orientada a tratar los problemas del cambio climático suele ser considerada una de las políticas ambientales más complejas de definir y de más difícil aplicación, debido a la gran cantidad de factores que intervienen en ese proceso, siendo uno de los más importantes la diversidad de intereses de los agentes implicados y la variedad de actitudes, valores y comportamientos de la población (Dessai, O'Brien y Hulme, 2007). Al aceptarse en términos científicos que al menos una parte de las modificaciones del clima se debe a la acción del hombre, el análisis de cómo valora la población la gravedad de tales cambios y de cómo se comporta ante ellos adquiere una notable relevancia para consensuar políticas o definir estrategias que sean realmente viables. Existe, por tanto, una mutua interacción entre, de un lado, el espacio político de actuación y, de otro, el ámbito de las percepciones, valores, actitudes y comportamiento de los individuos en materia de medio ambiente.

Sin embargo, analizar esa interacción no es tarea fácil. Incluso se ha apuntado en algunos estudios que resulta casi imposible aprehender en su totalidad las repercusiones del cambio climático en la actividad humana y el efecto de las acciones del hombre en este grave problema global (O'Riordan y Jordan, 1999). De ahí se deduce la necesidad de promover foros de interacción entre instituciones públicas y ciudadanía con objeto de intercambiar opiniones y debatir en común sobre temas tan complejos como los relacionados con el cambio climático. En recientes conferencias científicas, como la organizada por la Royal Geographical Society en agosto de 2007, se insiste en este aspecto y se recomienda prestar más atención al entorno social en el que se desarrollan las estrategias de mitigación y adaptación. Este punto de vista también ha sido recogido por una corriente de la literatura especializada (Tjernström, y Tietenberg, 
2008), y en buena medida también queda incorporado en el IV Informe de Evaluación del IPCC. En este último documento se afirma con claridad la importancia que tiene la participación de la sociedad civil en el proceso de toma de decisiones en este área de la política ambiental. Este planteamiento en favor de una amplia participación social en las políticas ambientales de escala global supera los anteriores enfoques, que se limitaban a impulsar sólo la colaboración de los actores directamente implicados en el desarrollo de tales políticas (Ministerio de Medio Ambiente, 2007). Se parte, por tanto, de la convicción de que la opinión pública en su conjunto es un aspecto esencial para vertebrar de un modo adecuado y eficiente las políticas sobre el cambio climático.

El objetivo de este artículo es avanzar en el análisis de la importancia que tiene la opinión pública en el tratamiento de los problemas globales relacionados con el medio ambiente, y contribuir a un mejor conocimiento del modo en que la población percibe tales problemas y valora las políticas ambientales, tomando como referencia una región, Andalucía, especialmente sensible al cambio climático. Andalucía es un área representativa del denominado déficit de implementación de la política europea de medio ambiente en el extremo sur de la UE (Liefferink, Lowe, Mol, 1993), siendo una región que goza de bastante autonomía en el desarrollo de sus propias políticas ambientales (Borzel, 2003). Asimismo, la región andaluza es un área significativa (en términos estrictamente físico-ambientales) de las repercusiones que tiene el proceso de cambio climático, debido a la relevancia de los efectos provocados por la alteración de las precipitaciones pluviométricas y los cambios en las temperaturas.

\section{Políticas AMBientales, OPINIÓN PÚBlica Y ESCALA REgIONAL/LOCAL}

En el estudio de las relaciones entre la opinión pública y las políticas ambientales se viene admitiendo desde hace tiempo que los esfuerzos en gasto público realizados por los gobiernos en este área se explican, en cierto modo, por la influencia que ejerce la población (Dunlap, 1995; Beatty, 1991). Se admite también que tal influencia se debe menos a la preocupación de la ciudadanía por el deterioro general del medio ambiente que a la importancia que le atribuye la población a temas ambientales específicos de especial sensibilidad, a escala local o regional y de fuerte incidencia en los comportamientos 0 estilos de vida (Barr, Gilg y Ford, 2003). Sin embargo, tanto la opinión pública como las políticas ambientales presentan variaciones importantes de unos países a otros y entre regiones dentro de un mismo país (Milfont, Dukitt y Cameron, 2006), variedad que es más acentuada en momentos, como ahora, de transición climática. A ello habría que añadir, tal como apunta una corriente de la Sociología Ambiental, que la opinión pública suele adaptarse en su vida cotidiana a los problemas ambientales de carácter global (el aumento de las temperaturas, los cambios en la estacionalidad del clima, las alteraciones de los periodos de lluvia, etc.), lo que reduciría su preocupación ante estos problemas y su disposición a actuar para mitigar sus efectos (Blake, 2001; Gooch, 1995; Scott y Willits, 1994; Carrus, Bonaiuto y Bonnes, 2005). 
Por ello, uno de los debates más relevantes en la política ambiental gira en torno al problema de definir la escala geográfica más idónea para aplicar las medidas de adaptación y mitigación ante el problema del cambio climático. Como se ha indicado, estas políticas pretenden resolver problemas de estricta naturaleza ambiental, pero su contenido y orientación están condicionados por el diseño político-institucional previo y por la propia opinión pública (Hannigan, 2006). Una notable corriente de opinión, no sólo en términos académicos, sino también institucionales, plantea que el nivel regional (e incluso el local) es la escala más adecuada para el diseño de una política ambiental de carácter participativo. La agregación de estrategias regionales o subregionales parece producir una mayor implicación de las poblaciones afectadas y, en consecuencia, permite establecer pautas más realistas de actuación (Vorkin y Riese, 2001; Ignatow, 2006; Paniagua, Moyano, 1998; Tabara, 2003, Yabar, 2006). En la Unión Europea, la escala regional ha sido considerada relevante para la definición y puesta en práctica de las políticas ambientales (Bodiguel y Buller, 1994; Buller, 1995), hasta el punto de que ya se ha utilizado en varios países (Buller, Lowe y Flynn, 1993).

Son numerosos los organismos internacionales y las organizaciones ecologistas que han señalado que una de las áreas geográficas sobre las que tendrá mayores efectos el cambio climático será el sur de Europa, y más concretamente, las regiones mediterráneas. De hecho, el informe del panel de la ONU predice que el sur de Europa es el área donde se concentrarán los efectos más negativos del calentamiento global, provocando veranos mucho más cálidos y menores precipitaciones pluviométricas, lo que dará como resultado una menor productividad agraria y una extensión notable del estrés hídrico (Karas, 1997). En ese contexto, Andalucía, que es la región más meridional y extensa del sur de Europa, sería una de las regiones más afectadas en el futuro por las variaciones climáticas.

Este punto de vista ha sido incorporado a la agenda política, al aceptarse que España es uno de los países más expuestos y vulnerables al cambio climático. En términos políticos, se ha reconocido incluso que el cambio climático tendrá en España más relevancia que en el resto de Europa (Narbona, 2005), opinión corroborada por las principales asociaciones ambientales de ámbito internacional que operan en España, como ADENA o Greenpeace. La importancia que se concede al cambio climático se pone de relieve en el papel de liderazgo que desempeña España, a nivel político, a la hora de asumir compromisos para paliar este problema en el escenario de la Unión Europea.

En ese contexto se formuló el Plan Nacional de Adaptación al Cambio Climático (Ministerio de Medio Ambiente, 2007). En dicho Plan se insiste en su carácter participativo, planteándose la necesidad de desarrollar políticas que promuevan entre la ciudadanía comportamientos adaptativos en materia de medio ambiente, partiendo de escenarios regionales o sub-regionales. Tal planteamiento se basa en la idea de que cada estilo de política ambiental responde a una combinación de intereses de tipo local o regional, ligados a la particular relación existente entre sociedad y medio ambiente en cada ámbito territorial (Kasapoglu y Ecevit, 2002). Se reconoce, así, que el comportamiento de la 
población se produce en el escenario político-institucional más cercano al territorio donde reside (Niemeyer, Petos y Hobson, 2005), un escenario sobre el que inciden los actores y organismos internacionales (Fisher, 2003). Además, diversos estudios ya apuntan a que muchos comportamientos pro-ambientales no se pueden poner en práctica debido a obstáculos y dificultades ligados al entorno físico más cercano al individuo (por ejemplo, carencia de suficientes contenedores para el reciclaje de residuos sólidos urbanos; falta de plantas de depuración de las aguas residuales; escasez de medios de transporte público, etc.), característica ésta que refuerza la relevancia de las políticas regionales/ locales a la hora de remover esos obstáculos (Slimak y Dietz, 2006).

Fruto de las directrices contenidas en el citado Plan, el Gobierno español puso en marcha la llamada Estrategia Española de Cambio Climático y Energía Limpia (EECCEL), que concede un papel relevante a los gobiernos de las Comunidades Autónomas en la elaboración de sus propias estrategias (dado que las competencias administrativas en relación con el medio ambiente les han sido totalmente transferidas). La Estrategia Andaluza ante el Cambio Climático se sitúa en ese marco de iniciativas regionales, presentando de forma articulada un conjunto de medidas intersectoriales, entre las que destacan las relacionadas con la prevención y el control de los problemas estrictamente ambientales, con la política forestal y la biodiversidad, así como con la mitigación y la adaptación a los impactos causados en el medio ambiente por el cambio climático (Junta de Andalucía, 2007).

\section{EL ECOBARÓMETRO dE ANDALUCía (EBA) COMO INSTRUMENTO dE ESCALA REGIONAL}

Habitualmente, los problemas ambientales se definen, caracterizan y construyen, no sólo sobre una base de naturaleza física (Jones, 2002; Jones y Dunlap, 1992), sino también a partir de cómo los percibe la población y de cómo se comportan, o pretenden comportarse, los individuos respecto a tales problemas (Sunderlin, 1995). Tal como indica el trabajo de Harrik y Jamieson (1995), las orientaciones políticas y el sistema de valores de la opinión pública son dos elementos fundamentales en la construcción y definición de los problemas ambientales y en su posible solución (Penning-Rowsell, Jonson y Tunstall, 2006). De ahí se deduce la necesidad de avanzar tanto en el análisis de la respuesta político-institucional a las incertidumbres que presenta el problema del cambio climático, como de las interacciones que se producen entre las acciones políticas y las preocupaciones de la población sobre este problema. Las políticas que, en relación con el cambio climático, aspiren a ser sostenibles en el largo plazo precisan de una modificación de las actitudes y comportamientos individuales, de tal modo que sólo serán factibles si las opiniones de los ciudadanos son tenidas en cuenta mediante procesos de participación (Lorenzoni, Nicholson-Cole y Whitmarsh, 2007; UNFCCC, 2004).

Aunque la presencia de instrumentos de participación en la puesta en marcha de las políticas ambientales se considera un asunto relevante (Lorenzoni y Pidgeon, 2005), este principio orientador está poco desarrollado todavía en España (Sunyer y Hueso, 
2004). A lo más que llegan tales procesos de participación es a realizar encuestas para medir las actitudes y comportamientos de una determinada población en relación con los temas ambientales. Aun reconociéndose el carácter restrictivo de este enfoque sobre la participación, es cierto que las encuestas de opinión pública constituyen una herramienta útil para conocer las singularidades de la población (y su evolución) en lo que se refiere al modo de percibir y valorar los procesos de cambio global, si bien son encuestas que se han venido realizando sobre una base nacional (Brechin, 2003). No obstante, diversos estudios regionales muestran que la opinión pública en estos asuntos varía notablemente de unos territorios a otros dentro de un mismo país, por lo que se hace necesario conocer tales variaciones geográficas a la hora de formular una adecuada política ambiental construida desde abajo (Harrington, 2001). Además, se indica que, a partir de la información proporcionada por los estudios de ámbito regional, es posible (re)articular en esta escala escenarios con un grado de libertad mayor que en la escala nacional (Jones, 2002).

Desde 2001, la Junta de Andalucía viene financiando la elaboración del Ecobarómetro de Andalucía (EBA), una encuesta de periodicidad anual realizada por el Instituto de Estudios Sociales Avanzados (IESA-CSIC), que permite conocer las actitudes y comportamientos de la población andaluza en materia de medio ambiente, así como la valoración que hacen los andaluces de las políticas desarrolladas por su gobierno regional. Este es el único caso en España donde se ha puesto en marcha un sistema anual de encuestas sobre medio ambiente, que posibilita la construcción de un sistema de indicadores sociales para medir la variación periódica de tales dimensiones de la conciencia ambiental entre la población de Andalucía.

Cada encuesta del EBA se realiza sobre una muestra representativa de la población andaluza de 1.500 individuos, con un nivel de error del 2,8\% y un nivel de confianza del 95\%. La encuesta se estructura en cuatro bloques: a) percepción social de la importancia que tienen los problemas ambientales en comparación con otros problemas, e identificación de los que más preocupan a los andaluces en los distintos ámbitos territoriales (local, regional, nacional y global); b) valoración de los agentes sociales y de las instituciones públicas que intervienen en la vertebración de las políticas ambientales; c) actitudes y comportamientos individuales y colectivos de los andaluces en relación con el medio ambiente; d) valoración de las distintas políticas ambientales desarrolladas por el gobierno regional. Para preparar el presente trabajo se han utilizado los EBA de 2001 a 2006 y los primeros resultados del año 2007, utilizándose principalmente las preguntas relativas a las actitudes de la población sobre el cambio global y su valoración de las políticas públicas.

El EBA parte de considerar las cuestiones ambientales como asuntos de interés público, es decir, como asuntos sobre los cuales los ciudadanos adoptan una determinada posición, que se considera, además, relevante para el diseño de las políticas públicas. De esta forma, el EBA pretende analizar las dimensiones en que se estructura la conciencia ambiental de la población, articulando el nivel afectivo y cognitivo con el de la conducta y el comportamiento. En definitiva, el EBA pretende analizar las dimensiones más usuales del comportamiento humano en relación con el medio ambiente y, además, estudiar 
cómo valoran los ciudadanos la gestión realizada por las instituciones públicas a través de la política medioambiental.

\section{Resultados del EBA SOBRE CAMBIO CLIMÁTICO Y POLítICAS AMBieNTALES}

En España, como en otros países de su entorno geográfico, el medio ambiente forma parte de la preocupación de los ciudadanos, si bien en un nivel comparativamente bajo respecto a otros problemas sociales o económicos. De acuerdo con la última encuesta de opinión disponible (n. ${ }^{\circ}$ 2728) del Centro de Investigaciones Sociológicas (CIS) de julio de 2007, los problemas ambientales sólo son citados como problemas importantes por el $2,7 \%$ de los españoles en una pregunta de respuesta abierta. Esta reducida percepción sobre la importancia relativa de los problemas relacionados con el medio ambiente se mantiene constante sin grandes alteraciones desde hace más de una década. El propio Ecobarómetro de Andalucía (EBA) también hace referencia a este asunto, apuntando una tendencia similar (Moyano y Jiménez, 2005).

\section{Percepción social de los problemas ambientales de escala global}

Aunque la preocupación general por las cuestiones ambientales se mantiene más o menos constante, los cambios más significativos se producen, sobre todo, por el valor diferente que le conceden a determinados problemas respecto a otros, según la escala territorial de referencia (Dunlap, 1995). Centrándonos en la escala global, el problema del agujero de la capa de ozono ha sido percibido por los andaluces como el primer problema del medio ambiente a nivel planetario en el periodo 2001-2006, pero ha sido superado por el cambio climático como primer problema en el EBA del 2007, donde casi la mitad de los andaluces han citado este problema como el que más les preocupa a escala global (ver Tabla 1).

El interés de la opinión pública andaluza en torno a este problema de escala global, cabe entenderlo en el marco de una más que notable preocupación en todos los países mediterráneos por los efectos de la actividad humana sobre el clima (EORG, 2002), al estar situados en una zona geográfica donde tales efectos (reflejados en la alteración de las temperaturas y la pluviometría) se manifiestan con gran nitidez (Niemeyer, Petos y Hobson, 2005). El EBA-2007 muestra que un 62,8\% de los andaluces (casi dos de cada tres) cree que el cambio climático es ya un problema actual, mientras que un 22,1\% lo percibe como un problema futuro. En general, el EBA-2007 nos dice que las personas más preocupadas por el medio ambiente y más comprometidas en preservarlo con su comportamiento, son también las que manifiestan índices más elevados de preocupación y conocimiento sobre el cambio climático.

Entre los principales efectos generales del cambio climático, los andaluces citan, sobre todo, el aumento de las temperaturas (uno de cada dos encuestados cita este efecto), el cambio en las precipitaciones pluviométricas (uno de cada tres) o la alteración 


\begin{tabular}{|c|c|c|c|c|c|c|c|c|c|c|c|c|c|c|}
\hline \multirow{8}{*}{ 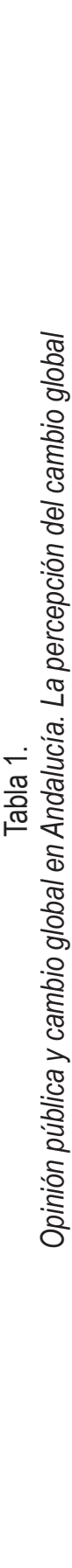 } & \multirow{8}{*}{ 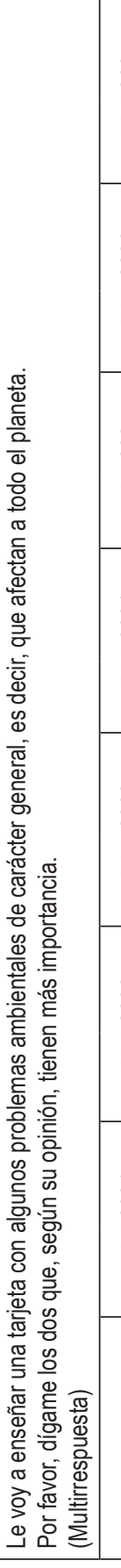 } & 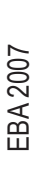 & 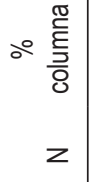 & $\begin{array}{l}\stackrel{\mathfrak{I}}{ \pm} \\
\stackrel{\rho}{\square}\end{array}$ & 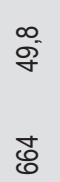 & $\begin{array}{l}\text { مू } \\
\text { న్ }\end{array}$ & $\begin{array}{l}\text { ণิ } \\
\text { চ্ল }\end{array}$ & 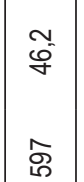 & ळi & $\underset{\square}{ }$ & $\stackrel{\sigma}{\sigma}$ & $\Re$ & న్ ̦̃ & \\
\hline & & 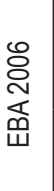 & 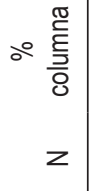 & $\begin{array}{l}\hat{\cong} \\
\stackrel{\cong}{\cong}\end{array}$ & $\begin{array}{l}\stackrel{\circ}{\Im} \\
\stackrel{্}{\circ}\end{array}$ & $\begin{array}{l}\text { స̃ } \\
\text { ᄋ্ন }\end{array}$ & $\begin{array}{l}\text { N } \\
\text { o } \\
\infty \\
\stackrel{8}{q}\end{array}$ & $\begin{array}{l}0 \\
\text { ơ } \\
\dot{\sigma}\end{array}$ & $\begin{array}{l}\text { న } \\
\text { ¿্ల }\end{array}$ & $E$ & স্ & $\begin{array}{l}r \\
\sim\end{array}$ & ֻి & \\
\hline & & 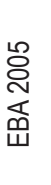 & 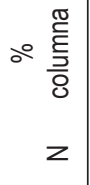 & $\begin{array}{l}\stackrel{+}{E} \\
\stackrel{g}{\leftarrow}\end{array}$ & 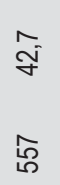 & $\begin{array}{l}\stackrel{\nabla}{\sim} \\
\stackrel{a}{N}\end{array}$ & 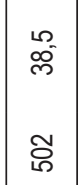 & 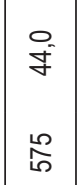 & চे & 음 & กิ & $\begin{array}{l}\digamma \\
-\end{array}$ & $\stackrel{\text { ஜn }}{\sim}$ & \\
\hline & & 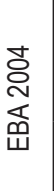 & 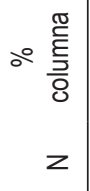 & $\begin{array}{l}\bar{\Xi} \\
\infty \\
\stackrel{\rho}{\square}\end{array}$ & $\begin{array}{l}\frac{g}{m} \\
\frac{\bar{g}}{\sigma}\end{array}$ & $\begin{array}{l}\stackrel{\text { న }}{ } \\
\stackrel{\infty}{\infty}\end{array}$ & $\begin{array}{l}\stackrel{m}{\sim} \\
\mathscr{\infty}\end{array}$ & $\begin{array}{c}\frac{6}{6} \\
\frac{1}{2}\end{array}$ & 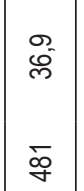 & $\stackrel{20}{\leftarrow}$ & N & & $\stackrel{\text { ஜn }}{\leftarrow}$ & \\
\hline & & 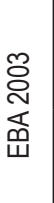 & 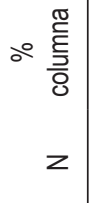 & 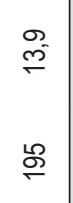 & $\begin{array}{l}\frac{0}{N} \\
\frac{10}{4}\end{array}$ & $\begin{array}{l}\stackrel{\infty}{\circ} \\
\stackrel{p}{\sigma}\end{array}$ & $\begin{array}{l}\stackrel{\infty}{\sim} \\
\stackrel{N}{\text { న్ }}\end{array}$ & 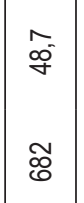 & $\begin{array}{l}\hat{\widetilde{\sigma}} \\
\frac{m}{6}\end{array}$ & $r$ & $\stackrel{\circ}{\circ}$ & $\nabla$ & $\stackrel{\text { ơ }}{\leftarrow}$ & \\
\hline & & 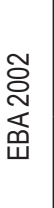 & 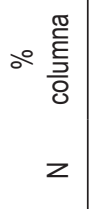 & $\begin{array}{l}\stackrel{+}{\mathscr{N}} \\
\stackrel{N}{*}\end{array}$ & $\begin{array}{l}\frac{6}{m} \\
\frac{5}{q}\end{array}$ & $\begin{array}{l}\bar{\sim} \\
\text { o } \\
\text { ờ }\end{array}$ & $\hat{\text { s }}$ & $\frac{m}{\infty}$ & $\begin{array}{l}\stackrel{\infty}{\leftarrow} \\
\stackrel{8}{8}\end{array}$ & $r$ & $\begin{array}{l}\infty \\
\omega^{\infty}\end{array}$ & 10 & $\stackrel{\substack{f \\
\leftarrow}}{-}$ & \\
\hline & & 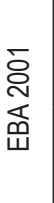 & 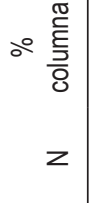 & $\begin{array}{l}\stackrel{\circ}{\oplus} \\
\stackrel{2}{\circ}\end{array}$ & $\begin{array}{l}\stackrel{2}{\text { fే }} \\
\text { 导 }\end{array}$ & $\begin{array}{c}\text { N } \\
\stackrel{\sim}{~} \\
\hat{ల}\end{array} \mid$ & $\begin{array}{l}\hat{\oplus} \\
\hat{\sigma}\end{array}$ & $\begin{array}{c}\stackrel{\rho}{\text { గె }} \\
\bar{\varnothing}\end{array}$ & $\begin{array}{l}\text { : } \\
\text { 해 }\end{array}$ & m & $\stackrel{\Re}{\stackrel{2}{2}}$ & $\sim$ & $\underset{\check{D}}{\leftarrow}$ & 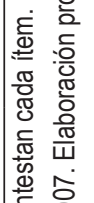 \\
\hline & & & & 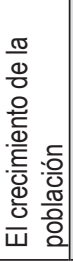 & 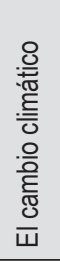 & 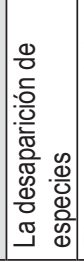 & 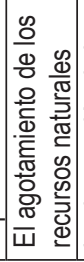 & 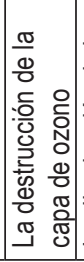 & 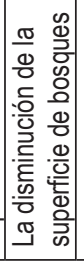 & 导 & z & z & 要 & 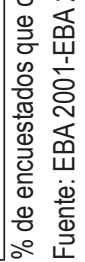 \\
\hline
\end{tabular}


de las estaciones climáticas durante el año (uno de cada cinco). Por el contrario, los efectos ambientales del cambio climático que no son percibidos a través de la experiencia individual, sino que requieren de una instancia mediadora para su conocimiento (como los efectos cada vez más dañinos de la desertificación, el deshielo de los polos, los incendios forestales 0 la desaparición de especies), son mencionados con bastante menos frecuencia por los andaluces (en un porcentaje siempre inferior al $5 \%$ de los encuestados).

No obstante, si se comparan los EBA de 2001 y 2007, observamos cómo se concentra el interés y la preocupación de la opinión pública andaluza en el problema del aumento de las temperaturas como principal efecto percibido del cambio climático, mientras que la disminución de las lluvias no es advertida como un efecto directo de este fenómeno de cambio global, sino que se asume como algo consustancial al clima mediterráneo. Tal conclusión tiene notables implicaciones en un área geográfica muy ligada al sector servicios de tipo turístico, lo que plantea la necesidad de una adecuada política de adaptación a los efectos del cambio climático.

\section{La escala territorial en la percepción social del cambio climático}

Que los ciudadanos reconozcan que existe un problema de cambio climático y estén informados de un modo general de los efectos no significa que les resulte fácil identificar sus efectos concretos en un determinado territorio, siendo esta dificultad un asunto de la máxima importancia en la investigación socioambiental destinada a orientar las políticas de mitigación o adaptación (Harrington, 2001) Se plantea así el problema de la escala territorial.

Tal dificultad estriba en el hecho de que no es fácil trasladar la gravedad de los problemas ambientales de carácter global a la conciencia de los habitantes que residen en un territorio determinado, ya que eso implica integrar tales problemas en perspectivas socioeconómicas concretas y en el sistema de valores éticos predominante en cada comunidad. Ante problemas ambientales cuya gravedad sea reconocida objetivamente en términos generales, puede ocurrir que en una determinada comunidad la población no la perciba así, o no quiera admitirlo, debido a razones socioeconómicas o simplemente a que se utilice una escala diferente de valores éticos. Por ejemplo, en el primer caso podemos encontrar una población que no admita la existencia de un problema grave de desertificación en un espacio territorial determinado de su comunidad, por no querer que se vean afectadas negativamente sus expectativas de desarrollo económico. En el segundo caso, podemos encontrar que en una determinada comunidad no se valore como intrínsecamente malo el riesgo evidente de extinción de ciertas especies animales (por ejemplo, el lince ibérico), y que, por tanto, la población no acepte que sea considerado un problema social relevante por sus efectos sobre la pérdida de biodiversidad, y en consecuencia no esté dispuesta a apoyar las medidas dirigidas a su protección. Todo ello puede tener notables repercusiones en la valoración social que reciben las políticas ambientales y los organismos que las desarrollan, valoración que 
dependerá de la escala en la que cada institución desarrolla sus actuaciones en esta materia.

Esto es así porque las políticas puestas en marcha por las instituciones de ámbito regional o local, por ejemplo, suelen ser percibidas por la población como relevantes sólo para la resolución de problemas ambientales en esa escala geográfica, ya que, entienden, no pueden contribuir significativamente a la solución de los problemas ambientales de escala global. Son, sin embargo, las políticas desarrolladas en los niveles nacional o internacional y las instituciones responsables de aplicarlas, las que son percibidas por los ciudadanos como las más idóneas para paliar los problemas relacionados con el medio ambiente a escala global. La población asocia, por tanto, la solución de los problemas de ámbito global a las instituciones que actúan en esa escala, y circunscriben las acciones de las instituciones regionales y locales a la gestión y tratamiento de los problemas específicos que se manifiestan en esos ámbitos. A los ciudadanos no les resulta fácil asociar problemas globales y acciones regionales/locales, lo que constituye un problema para las políticas que tratan de paliar los efectos del cambio climático y que, como se ha señalado, buscan la implicación de la ciudadanía.

Tomando como referencia los EBA, se confirma esta tesis, existiendo una clara asociación positiva entre la escala de los problemas ambientales y el ámbito de actuación de las instituciones. En efecto, según los datos de los EBA, la población andaluza atribuye a la Unión Europea la mayor responsabilidad en la resolución de los problemas globales (cambio climático, pérdida de biodiversidad, desertificación, disminución de masas forestales, etc.). Este orden cambia cuando se les pregunta por problemas regionales/locales (por ejemplo, el agua, el ruido, la suciedad de las calles, los incendios forestales, la contaminación de ríos y mares, la contaminación atmosférica, etc.), siendo en ese caso los ayuntamientos y la Junta de Andalucía las instituciones a las que se les atribuye la mayor responsabilidad.

Con la información proporcionada por los EBA hemos profundizado algo más en el análisis de estos asuntos, centrándonos en la escala regional. Lo primero que se observa es la consistencia en la valoración que hacen los andaluces sobre las instituciones regionales en materia de medio ambiente y sobre las distintas políticas que desarrollan, recibiendo valoraciones similares. Esta consistencia de los valores medios es una de las principales características de la opinión pública y las políticas ambientales a nivel regional en Andalucía (Tabla 2). Ello muestra que los andaluces apenas discriminan entre las distintas políticas ambientales de ámbito regional, sino que las valoran en general desde una perspectiva de conjunto, sin tener en cuenta si esas políticas contribuyen en mayor o menor medida a la solución de los problemas ambientales a los que van dirigidas.

Un paso más en el análisis sería preguntarse si la preocupación por el problema del cambio climático hace variar o no el modo como valoran los ciudadanos las distintas políticas ambientales desarrolladas por las instituciones regionales (es decir, por la Junta de Andalucía). Se ha analizado con datos del EBA 2007 la valoración que hacen los andaluces de las políticas ambientales regionales, comparando cómo valora esas políticas la población que expresa alta preocupación por el cambio climático, y 


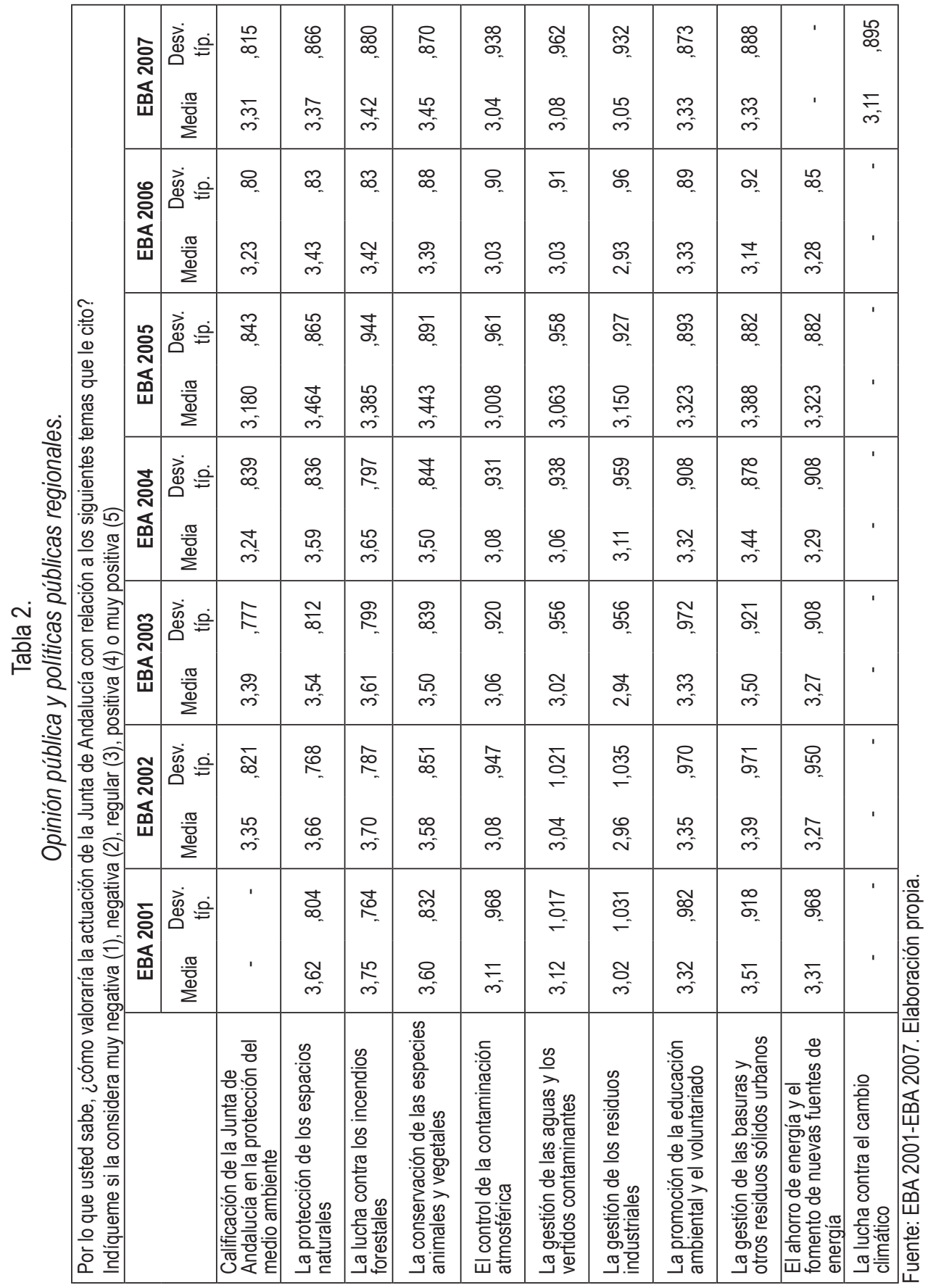




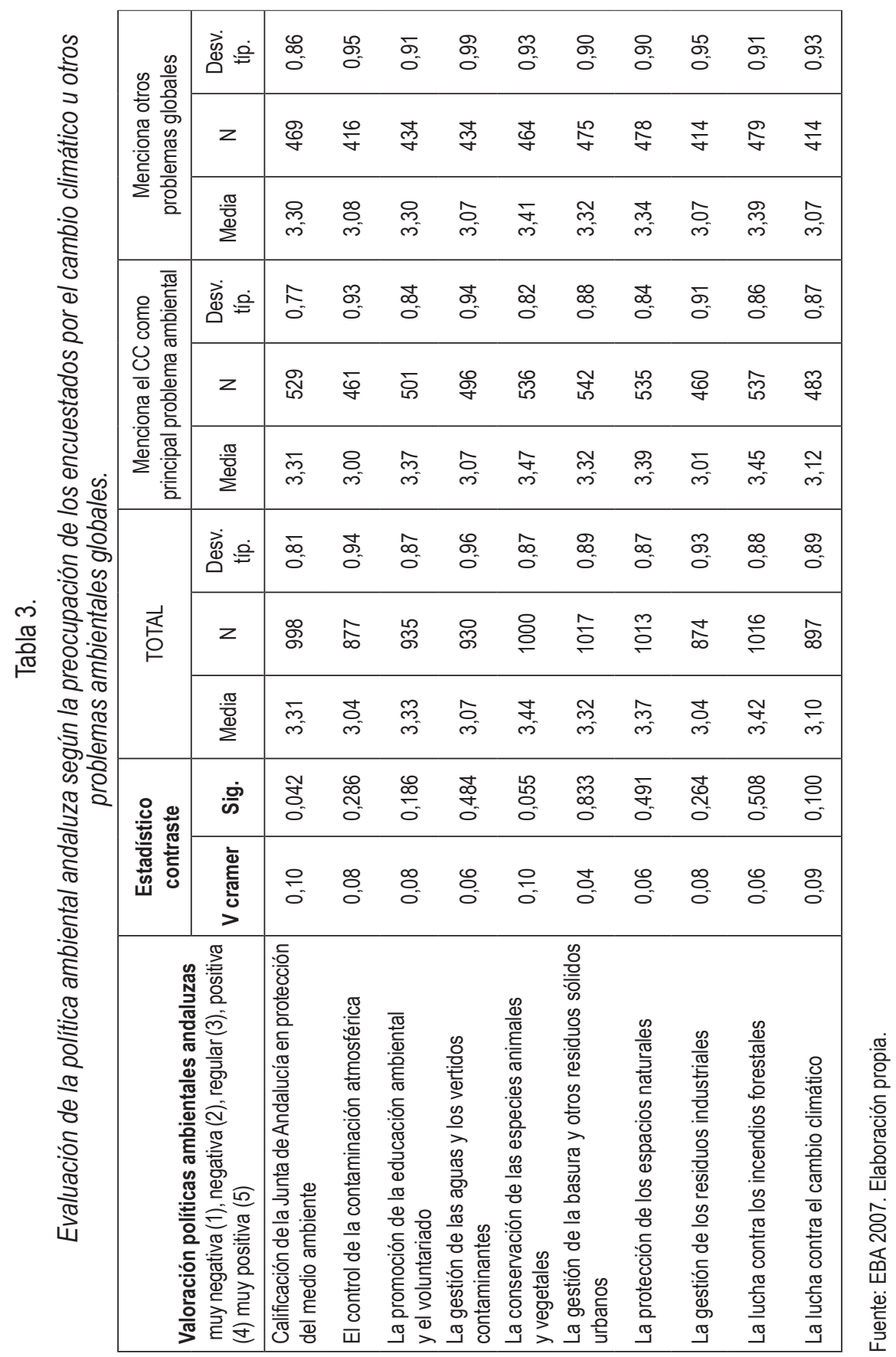


la que se preocupa por otros problemas ambientales. No existe entre ambos grupos una clara diferencia en la valoración de la política ambiental de la Junta de Andalucía en su conjunto, expresando ambos una posición intermedia (Tabla 3). En el caso de actuaciones o políticas ambientales específicas, tampoco se observan entre estos dos grupos diferencias significativas.

Estos resultados sugieren, de alguna forma, cierta independencia entre, de un lado, la preocupación general de los andaluces por los problemas globales del medio ambiente, y, de otro, su valoración de las políticas regionales. Todo parece confirmar la tesis antes señalada, a saber: que los andaluces no perciben todavía con claridad que la lucha contra los efectos del cambio climático sea una combinación de políticas sectoriales que se aplican a nivel regional o local. Para los andaluces la solución de los problemas ambientales de escala global pertenecería a un ámbito donde se precisan consensos que exceden el ámbito regional o local. En consecuencia, a la hora de valorar la necesidad de aplicar medidas de mitigación y adaptación al cambio climático, los andaluces las circunscriben al ámbito global, sin conectarlas con las acciones de ámbito regional o local, dominando, por tanto, en la opinión pública de Andalucía una perspectiva vertical (de arriba-abajo). Es posible argumentar, en tal sentido, que los problemas ambientales de escala global son considerados por los andaluces como objeto de una especie de "suprapolítica" que se define en instancias globales (europeas, internacionales,...) y que no guarda relación alguna con las políticas de escala regional/local. Para ellos, las acciones que se aplican en el ámbito regional/local responden al objetivo de resolver problemas ambientales específicos, es decir, los que se manifiestan de forma concreta en los territorios más cercanos, sin plantearse que tales acciones puedan contribuir a la lucha contra los efectos del cambio climático y a promover un desarrollo sostenible a nivel planetario.

\section{Conclusiones}

Como indica la literatura especializada, las actitudes y los comportamientos en relación con el cambio climático son notablemente complejos (O'Connor, Bord, Fisher, 1999). Este trabajo trata de contribuir a un aspecto que tiene cada vez más relevancia, a saber: la importancia de los escenarios regionales en la interacción entre opinión pública y políticas destinadas a mitigar los efectos del cambio climático.

El caso de la política ligada al cambio climático es interesante de analizar en el caso de los países del sur de Europa, ya que es un tipo de política tradicionalmente caracterizada por su carácter subsidiario de los intereses ambientales del norte de Europa (Pridham y Konstadakopulos, 1997). Entre los andaluces, los problemas ligados al cambio climático se han convertido de forma paulatina, pero en un corto periodo de tiempo, en la primera preocupación ambiental de escala global, coincidiendo con 
el desarrollo de estrategias políticas y actuaciones públicas para la mitigación de sus efectos. En todo caso, la consolidación del cambio climático como primera preocupación ambiental de la población andaluza está ligada a la preocupación que genera en la región problemas concretos, tales como el ascenso de las temperaturas, la alteración de las precipitaciones pluviométricas, el avance de los procesos de desertificación o la ya visible erosión de los suelos.

Pero quizás uno de los resultados más interesantes del análisis realizado a partir de los datos proporcionados por los EBA sea su contribución al debate sobre si las acciones regionales son, o no, importantes en el desarrollo de políticas dirigidas a la resolución de problemas ambientales de escala global. Si bien la tendencia dominante en la literatura especializada parece apuntar a que la población valora la importancia de las políticas regionales/locales, en el caso de Andalucía esto no es tan evidente. La opinión pública separa con bastante claridad la responsabilidad de las distintas instituciones en la solución de los problemas ambientales según su escala geográfica (locales, regionales, nacionales y europeos), y todo ello en una especie de gradación donde no aprecian interrelación alguna entre ellas. Esto muestra el predominio en la opinión pública andaluza de una perspectiva vertical (de arriba hacia abajo) a la hora de percibir las estrategias más idóneas para afrontar la lucha contra el cambio climático, excluyendo a las instituciones regionales/locales a la hora de asignar responsabilidades en la mitigación de los problemas globales.

En la literatura especializada (Cohen, 1998) se ha sugerido que los problemas ambientales ligados al cambio climático modifican los esquemas clásicos sobre las percepciones y actitudes ambientales de la población, que han inspirado las políticas de desarrollo sostenible. La idea de que, en materia de desarrollo sostenible, la población percibe con claridad los efectos globales de las acciones y comportamientos que se realizan a nivel regional/local y valora la necesidad de combinar políticas globales y regionales/locales, no parece que se cumpla en el caso de los problemas relacionados con el cambio climático.

Esto es lo que se confirma en el estudio referido a Andalucía. El EBA señala que la población atribuye la mayor responsabilidad en la mitigación de los efectos producidos por el cambio climático a las instituciones de mayor escala geográfica, excluyendo a las instituciones regionales/locales. Los andaluces confieren protagonismo a las políticas regionales sólo en lo que se refiere a los problemas de "su" medio ambiente, sin ponerlas en relación con los posibles efectos que pudieran tener en la gestión de los problemas globales. Esta conclusión tiene implicaciones no sólo para el debate académico (que es el principal objetivo de este artículo), sino también para el debate político, donde parece necesario, a la luz de nuestros resultados, que se haga ver la opinión pública que el cambio climático es un problema global, pero que su resolución ha de ser el resultado de la combinación de acciones públicas y comportamientos sociales que comienzan en el ámbito territorial más cercano al ciudadano. 


\section{REFERENCIAS BibLIOGRÁFICAS}

Barr, S., A. Gilg y N. Ford. 2003. "Environmentalism in Britain today. Who are environmentalists?". Town and Country Planning: 185-186.

Beatty, K. M. 1991. "Public opinion data for environmental decision making: the case of Colorado Springs". Environment Impact Assessment Review 11: 29-51.

Blake, D. E. 2001. "Contextual effects on environmental attitudes and behaviour". Environment and Behavior 33:708-725.

Bodiguel, M. y H. Buller. 1994. "Environmental policy and the regions in France". Regional Politics and Policy 4: 92-109.

Borzel, T. A. 2003. Environmental leaders and laggards in Europe. Why there is (not) a 'Southern Problem'. Londres: Ashgate.

Brechin, S. R. 2003. "Comparative public opinion and knowledge on global climatic change and the Kyoto Protocol: the US versus the world?". International Journal of Sociology and Social Policy 23: 106-134.

Buller, H. 1995. "Local actors, national stage, European theatre: Europeanization, nationalization and localization in European community environmental policy". Ponencia presentada al ECPR Joint Sessions. Burdeos: France.

Buller, H., Ph. Lowe y A. Flynn. 1993. "National responses to the Europeanisation of environmental policy: a selective review of comparative research". En Liefferink, J. D., Ph. Lowe y P. J. Mol (eds.) European integration and environmental policy. Londres: Belhaven Press.

Carrus, G., M. Bonauto y M. Bonnes. 2005. "Environmental concern, regional identity, and support for protected areas in Italy". Environment and Behavior 37: 237-257.

Cohen, S., D. Demeritt, J. Robinson y D. Rothman. 1998. "Climate change and sustainable development: towards dialogue". Global Environmental Change 8:341-371.

Dessai, S., K. O'Brien y M. Hulme. 2007. "Editorial: on uncertainty and climate change". Global Environmental Change 17: 1-3.

Dunlap, R. E. 1995. "Public opinion and environmental policy". In Lester, J. P. (ed.). Environmental Politics and Policy. Theories and Evidence, pp. 64-114. Durham: Duke Univ. Press.

EORG-The European Opinion Research Group. 2002. The attitudes of Europeans towards the environment. Eurobarometer 58.0.

Fisher, D. R. 2003. "Global and domestic actors within the global climate change regime: toward a theory of the global environmental system". International Journal of Sociology and Social Policy 23: 5-30.

Gooch, G. D. 1995. "Environmental beliefs and attitudes in Sweden and the Baltic states". Environment and Behaviour 27: 513-539. 
Hannigan, J. A. 2006. Environmental sociology. A social constructionist perspective. Londres: Routledge. (First Edition 1995).

Harrington, L. M. B. 2001. "Attitudes toward climate change: major emitters in southwestern Kansas". Climate Research 16: 113-122.

Harrick, Ch. y D. Jamieson (1995. "The social construction of acid rain. Some implications for science/policy assessment". Global Environmental Change 5:105-112.

Ignatow, G. 2006. "Cultural models of nature and society. Reconsidering environmental attitudes and concern". Environment and Behaviour 38: 441-461.

IPCC. 2007. Climate Change 2007: Synthesis Report. Contribution of Working Groups I, II and III to the Fourth Assessment Report of the Intergovernmental Panel on Climate Change [Core Writing Team, Pachauri, R. $\mathrm{K}$ and A. Reisinger (eds.)]. IPCC. Geneva: Switzerland.

Jones, R. E. y R. E. Dunlap. 1992. "The social bases of environmental concern: have they changed over time?". Rural Sociology 57: 28-47.

Jones, S. 2002. "Social constructionism and the environment: through the quagmire". Global Environmental Change 12: 247-251.

Junta de Andalucía. 2007. Estrategia andaluza ante el cambio climático. Sevilla: mecanografiado.

Karas, J. 1997. El cambio climático en la región mediterránea. Barcelona: Greenpeace.

Kasapoglu, M. A. y M. C. Ecevit. 2002. "Attitudes and behaviour toward the environment. The case of Lake Burdur in Turkey". Environment and Behaviour 34: 363-377.

Liefferink, J.D., P. D. Lowe y A. P. J. Mol (eds.). 1993. European integration and environmental policy. Londres: Belhaven Press.

Lorenzoni, I., S. Nicholson-Cole and L. Whitmarsh. 2007. "Barries perceived to engaging with climate change among the UK public and their policy implications". Global Environmental Change, doi 10.1016/j. gloenvcha.2007.01.004.

Lorenzoni, I., N. Pidgeon and R. O'Connor. 2005. "Dangerous Climate Change: the role for risk research". Special issue of Risk Analysis 25(6):1387-1398, doi: 10.1111/j.1539-6924.2005.00686.x

Ministerio de Medio Ambiente. 2007. Plan nacional de Adaptación al Cambio Climático (PNACC). Madrid: Oficina Española del Cambio Climático.

Ministerio de Medio Ambiente. 2007. Estrategia Española de Cambio Climático y Energía Limpia. Horizonte 2007-2012-2020(EECCEL): Madrid.

Milfont, T. L., J. Duckitt y L. D. Cameron. 2006. "A cross-cultural study of environmental motive concerns and their implications for proenvironmental behaviour". Environment and Behavior 38: 745-767.

Moyano, E. y M. Jiménez. 2005. Los andaluces y el medio ambiente. Sevilla: IESA-CSIC y Junta de Andalucía. 
Narbona, C. 2005. "El cambio climático en España es mayor que en el resto de Europa". Actualidad Ciencia y Tecnología. http//actualidad.terra.es/artículo/html/av2146760

Niemeyer, S., J. Petts y K. Hobson. 2005. "Rapid climate change and society: assessing responses and thresholds". Risk Analysis 25:1443-1456.

O'Connor, R, E., R. J. Bord y A. Fisher. 1999. "Risk perceptions, general environmental beliefs, and willingness to address climate change". Risk Analysis 19: 461-471.

O'Riordan, T. y A. Jordan. 1996. "Social institutions and climate change", en O'Riordan, T. and J. Jager (eds.. Politics of climate change. An European perspective. pp. 65-105. Londres: Routledge.

O'Riordan, T. y A. Jordan. 1999. "Institutions, climate change and cultural theory: towards a common analytical framework". Global Environmental Change 9: 81-93.

Paniagua, A. y E. Moyano. 1998. "Medioambiente, desarrollo sostenible y escalas de sustentabilidad". Revista Española de Investigaciones Sociológicas: 151-178.

Penning-Rowsell, E., C. Johnson y S. Tunstall. 2006. "Signals' from pre-crisis discourse: Lessons from UK flooding for global environmental policy change?". Global Environmental Change 16: 323-339.

Pettenger, M. E. 2007. The social construction of climate change. Londres: Ashgate.

Pridham, G. y D. Konstadakopulos. 1997. "Sustainable development in Mediterranean Europe? Interaction between European, national and sub-national levels". En Baker, S., M. Kousis, D. Richardson y S. Young (eds.). The politics of sustainable development. Theory, policy and practice within the European Union, pp. 127-151. London: Routledge.

Scott, D. y F. K. Willits. 1994. "Environmental attitudes and behaviour. A Pennsylvania survey". Environment and Behavior 26: 239-260.

Seacrest, S., R. Kuzelka y R. Leonard. 2000. "Global climate change and public perception: the challenge of translation". Journal of the American Water Resources Association 36: 253-263.

Slimak, M. W., T. Dietz. 2006. "Personal values, beliefs, and ecological risk perception". Risk Analysis 26:1689-1705.

Sunderlin, W.D. 1995. "Global environmental change, sociology, and paradigm isolation". Global Environmental Change 5: 211-220.

Sunyer, C. y K. Hueso. 2004. "National Report. SOBIO. Mobilising the European social research potential in support of biodiversity and ecosystem management" Terra, 71 pp. (mimeo).

Tabara, D. 2003. "Spain: words that succeed and climate policies that fail". Climate Policy 3: 19-30.

Tjernström, E. y T. Tietenberg. 2008. "Do differences in attitudes explain differences in national climate change policies?". Ecological Economics 65: 315-324.

UNFCCC. 2004. Convención marco de la Naciones Unidas sobre el Cambio Climático. Bonn: Secretaria del Cambio Climático. 
Vorkin, M. y H. Riese. 2001. "Environmental concern in a local context". Environment and Behavior 33: 249-263.

Yabar, A. 2006. "Mecanismos de consulta, participación y cooperación para una estrategia española de cambio climático", $M+A$, Revista electrónica de medioambiente, 2. http://www.ucm.es/info/iuca/2006\%20 $2 \% 20$ yabar.pdf

EDUARDO MOYANO es Profesor de Investigación del Consejo Superior de Investigaciones Científicas (CSIC) y ocupa el cargo de Director del Instituto de Estudios Sociales Avanzados (IESA). Sus trabajos de investigación versan sobre acción colectiva y articulación de intereses, con especial referencia a las organizaciones de naturaleza representativa (sindicatos, asociaciones patronales y profesionales, federaciones de cooperativas, etc.). Asimismo, trabaja en temas de participación social, medio ambiente y desarrollo local.

ANGEL PANIAGUA es Investigador Científico del CSIC especializado en Geografia Rural y Sociología Ambiental, es autor de más de 100 publicaciones en las principales revistas nacionales e internacionales. Ha realizado investigaciones financiadas por importantes organismos nacionales y europeos.

REGINA LAFUENTE es Licenciada en Ciencias Políticas y Sociología por la Universidad de Granada y especialista en Investigación Social Aplicada y Análisis de Datos por el Centro de Investigaciones Sociológicas (CIS). Desde el año 2004 desarrolla su labor de investigación en el Instituto de Estudios Sociales Avanzados (IESA-CSIC). Sus temas de investigación se centran en el estudio de la conciencia ambiental y la participación social en cuestiones ambientales. Actualmente es la Directora Técnica del Ecobarómetro de Andalucía, proyecto que analiza las actitudes y comportamientos relacionados con el medio ambiente entre la población andaluza.

RECIBIDO: 23/01/08

ACEPTADO: 26/06/08

Publicado on-line: 15/06/09

RIS, VOL. 67, No 3, SEPTIEMBRE-DICIEMBRE, 681-699. ISSN: 0034-9712 